\title{
La indagación científica como enfoque pedagógico: estudio sobre las prácticas innovadoras de docentes de ciencia en EM (Región de Valparaíso)*
}

\begin{abstract}
Approach to inquiry as orientation to science teaching: a study about innovative practices of science teachers in secondary education classrooms of the Region of Valparaiso, Chile

Aproximação à Indagação Científica como Enfoque Pedagógico: Estudo sobre as práticas inovadoras de docentes de ciências, em aulas de educação média/secundária, na Região de Valparaíso, Chile
\end{abstract}

\section{Corina González-Weil, Mónica Cortéz, Paulina Bravo, Yasnina Ibaceta, Karen Cuevas, Paola Quiñones, Joyce Maturana \& Alejandro Abarca ${ }^{\text {a }}$}

aPontificia Universidad Católica de Valparaíso, Av. Universidad 330, Curauma, Valparaíso. Telf.: 0322274859. Correo electrónico: corina.gonzalez@ucv.cl

\section{RESUMEN}

El presente trabajo refiere los resultados de un estudio cualitativo cuyo objetivo fue describir y comprender las prácticas de seis docentes de ciencia en Educación Secundaria que han logrado transformar su enseñanza, aproximándose a un enfoque indagatorio. El enfoque metodológico empleado fue Grounded Theory, el cual se aplicó a la observación de 54 horas (cr.) de clase. El estudio aporta con información descriptiva y comprensiva sobre las características de las prácticas observadas, así como las condiciones que las permiten y las consecuencias que conllevan. Se finaliza discutiendo el impacto que este estudio tiene sobre la formación inicial y continua de docentes de ciencia de Educación Secundaria.

Palabras clave: educación en ciencias, indagación científica, prácticas pedagógicas, educación secundaria, competencia científica.
\end{abstract}

\begin{abstract}
This research refers the results of a qualitative study which purpose was to describe and understand the teaching practices of six secondary science teachers who have transformed their teaching practice by applying an inquiry approach. The method used was Grounded Theory applied to the observation of 54 class hours (cr.). This research contributes with descriptive and comprehensive information about characteristics of the observed practices, as well as the conditions that allow them and their consequences. Finally, we discuss the implications that this study has on pre-service and in-service secondary science teacher education.
\end{abstract}

Key words: science education, scientific inquiry, teacher practices, secondary education, scientific competence.

\section{RESUMO}

O presente trabalho mostra os resultados de um estudo qualitativo, cuja finalidade é descrever e compreender as práticas de ensino de seis professores de ciências secundários, que vieram a transformar seus métodos de ensino,

* $\quad$ Este trabajo se desarrolla y ha sido financiado en el marco del proyecto FONDECYT No ${ }^{\circ} 1080267$. Agradecemos a CONICYT, a la Dirección de Investigación e Innovación de nuestra Universidad y a los establecimientos, docentes y estudiantes que participaron de este estudio por el apoyo prestado. 
aproximando-os de uma técnica de questionamento, indagação. O método usado foi o Grounded Theory, aplicado à observação de 54 horas de aulas(cr.). Esta pesquisa contribui com informação descritiva e detalhada sobre características das práticas observadas, assim como as circunstâncias que as permitiram e suas conseqüências. Finalmente, nós discutimos as implicações que este estudo tem na formação inicial e contínua de professores de ciências de nível médio/secundário.

Palavras chave: educação científica, indagação científica, práticas de ensino, educação secundária, competência científica.

\section{INTRODUCCIÓN}

La Ciencia y la Tecnología son pilares del desarrollo social y económico de los países. Así lo han consensuado científicos, educadores y políticos, quienes argumentan que gracias a la generación de conocimiento y la transferencia de éste a la tecnología, se genera innovación y, en consecuencia, productividad. Adicionalmente, la ciencia constituye un eje estratégico del desarrollo humano, ya que implica no sólo el fortalecimiento de la capacidad crítica de una sociedad (Alberts, 2008; Láscaris, 2008), sino también una contribución a la inclusión y equidad social, bajo la idea de que en la actualidad el contar con competencia científica -entendida como un conjunto de conocimientos, capacidades y actitudes científicas- permite una mejor comprensión del medio y el poder participar de manera fundamentada en la sociedad (Macedo y Katzkowicz, 2005). Bajo este contexto, la educación científica tiene una importancia crítica no sólo para la Ciencia, también para el Mundo, convirtiéndose en uno de los pilares de la transformación de nuestras sociedades, contribuyendo a la equidad, la educación y la cultura (Reimí, 2002, Alberts, 2008).

Si bien la enseñanza de las ciencias evidentemente debe abordarse en todos los niveles educativos, según Gil y Vilches (2001) es la educación secundaria la etapa fundamental para plantear la alfabetización científica de los futuros ciudadanos y ciudadanas. Lamentablemente, y en general, la formación científica del alumnado en el sistema escolar chileno no está a la altura de este desafío.

Un informe de la Academia Chilena de Ciencias (2005) indica que en este nivel la formación científica del alumno "ha girado tradicionalmente en torno de una enseñanza desagregada o disciplinaria del saber científico, una instrucción enciclopedista, un aprendizaje memorístico de conocimientos atomizados, datos fragmentarios e informaciones puntuales, con una comprensión de la ciencia descontextualizada del mundo cotidiano y de las necesidades de la vida social" (Albertini et al., 2005: 46), diagnóstico que coincide con el análisis de otros autores acerca de la enseñanza en este nivel educativo (Chacón 2003; Pasmanik et al., 2005). A partir de este panorama, se hace indispensable una transformación en las prácticas de enseñanza de las ciencias, que estén orientadas a entregar a los alumnos un rol mucho más protagónico, en donde el aprendizaje es entendido como un proceso activo en el cual la exploración, la reflexión y la resolución de problemas ocupan lugares centrales (Devés, 2004).

Una manera innovadora de concebir la enseñanza de las ciencias se relaciona con el concepto de indagación científica. En el ámbito de la educación en ciencias el término "indagación" es a menudo entendido como uno de los objetivos de aprendizaje o, más comúnmente, como una metodología de enseñanza. Existen, en esta perspectiva, diversas definiciones para este concepto. Así, por ejemplo, Windschitl define indagación científica como un proceso en el cual "se plantean preguntas acerca del mundo natural, se generan hipótesis, se diseña una investigación, y se colectan y analizan datos con el objeto 
de encontrar una solución al problema" (Windschitl, 2003: 113), entendiendo que este proceso estaría presente tanto en el trabajo de generación de conocimiento científico, a nivel de comunidad científica, como en la generación de conocimiento científico escolar, a nivel de aula. Más concretamente aun, y refiriéndose específicamente a la actividad de aula, un proceso de indagación científica implicaría el "hacer observaciones, exhibir curiosidad, definir preguntas, recopilar evidencia utilizando tecnología y matemáticas, interpretar resultados utilizando conocimientos que derivan de investigación, proponer posibles explicaciones, comunicar una explicación basada en evidencia y considerar nuevas evidencias" (Programa ECBI, ${ }^{1}$ 2007). En cualquier caso, se trata de una enseñanza centrada en el alumno, en donde el docente orienta la construcción de conocimientos científicos en el alumnado a través de actividades concretas que involucran el poner en juego una serie de competencias relacionadas con el quehacer científico. No obstante lo anterior, y desde una perspectiva sociocultural, la indagación también puede entenderse como un enfoque pedagógico, es decir, una orientación hacia la reflexión en el proceso de enseñanza de las ciencias, (Abell et al., 2006) en el entendido de que es el docente quien indaga sus propias prácticas, para luego trasladar este proceso reflexivo y de indagación a la construcción de conocimiento científico por parte de sus alumnos. Esto implica, por parte del docente, una cierta "actitud indagatoria" hacia su propia vida, donde éste se concibe no sólo como un "enseñante", sino también como un aprendiz permanente, capaz de reflexionar acerca de su quehacer y transformarlo para su mejora, generando a su vez un conjunto de conocimientos y creencias que guían su quehacer en el aula.

Si bien podemos suponer que la mayoría de los docentes actualmente en ejercicio, fueron formados bajo un paradigma más bien tradicional de enseñanza, existen quienes a pesar de ello presentan una "actitud indagatoria" frente a sus prácticas, y han logrado transformarlas hacia un paradigma más constructivista, centrado en el alumno y obteniendo mejores resultados de aprendizaje. Pero, ¿qué hacen concretamente estos docentes en el aula? A pesar de que desde la teoría muchas son las características que se le atribuyen a una "buena enseñanza" de las ciencias (las que son descritas principalmente en la educación primaria), son escasos los estudios que describen, desde la evidencia, cómo esta enseñanza podría llevarse a cabo, menos aún en nuestro país. De allí que, en un artículo anterior (González-Weil et al., 2009), finalizamos planteando lo importante que sería investigar acerca de las prácticas de docentes secundarios de ciencia que, por propia iniciativa, se atrevían a traspasar las fronteras de la enseñanza tradicional de la ciencia, innovando y aproximándose a un enfoque indagatorio en sus prácticas. Con el objetivo de aprender de dichas experiencias docentes, el presente trabajo investiga desde un enfoque cualitativo las características de las prácticas de seis docentes secundarios de ciencia que, inmersos en un sistema tradicional, han logrado transformar sus prácticas aproximándose a un enfoque indagatorio de su enseñanza. Las preguntas que orientaron esta investigación fueron: ¿Qué actividades se realizan en la sala de clases y cómo se estructuran? ¿Qué ámbitos de competencia científica aborda el docente en su clase (capacidades, conocimientos, actitudes)? ¿Qué características tiene la interacción profesor-alumno y de qué manera apoya esta interacción el aprendizaje? Asumiendo la relación dialéctica entre prácticas y creencias el estudio explora las concepciones, fundamentaciones y elementos de la formación que subyacen a estas prácticas innovadoras y que en alguna

1 ECBI: Educación en Ciencias Basada en la Indagación. 
medida constituyen condiciones antecedentes que facilitan la realización de las prácticas observadas. En último término, se esbozan algunas reflexiones acerca de las enseñanzas que el estudio de prácticas innovadoras puede dejarnos para la transformación de la formación docente inicial y continua de los profesores de ciencia secundarios.

\section{METODOLOGÍA}

(a) Enfoque: El enfoque empleado en la presente investigación corresponde a la Teoría Fundamentada (Grounded Theory), la cual permite el desarrollo de un conjunto de conceptos integrados ofreciendo una explicación teórica detallada y precisa del fenómeno en estudio, trascendiendo del nivel meramente descriptivo y generando modelos explicativos del objeto de investigación (Strauss \& Corbin, 1990).

(b) Participantes: Los resultados presentados se refieren a las prácticas y creencias de seis docentes de la Región de Valparaíso, pertenecientes a Establecimientos Municipales (1), subvencionados (4) y particulares (1), de química (2), biología (2) y física (2), que presentaban, según un estudio anterior, ${ }^{2}$ procesos de implementación sistemática en sus innovaciones pedagógicas, caracterizados por una reflexión permanente sobre sus prácticas, lo que podría entenderse como una mayor cercanía a la indagación como enfoque pedagógico. Adicionalmente, en el contexto del mismo estudio anterior y en comparación con los alumnos de otro grupo de docentes secundarios de ciencia, los alumnos del grupo de docentes involucrados en este estudio, destacaban por obtener niveles significativamente superiores de competencia científica, al comparar entre niveles socioeconómicos similares. ${ }^{3}$

(c) Recolección de datos: Los resultados de este estudio se basaron en las observaciones de aprox. 54 horas cr. de clase (6 a 8 sesiones de clase por docente), mediante el registro audiovisual de éstas, complementadas con registros etnográficos de observadores in situ. Adicionalmente, se realizaron entrevistas de argumentos prácticos ${ }^{4}$ a 3 de los 6 docentes, y entrevistas abiertas sobre concepciones pedagógicas a 5 de los 6 docentes del grupo, de modo de apreciar la relación entre creencias y prácticas docentes.

(d) Análisis de datos: Los resultados obtenidos en el estudio son de tipo descriptivo, analítico interpretativo y explicativo. De acuerdo a los procedimientos determinados por la Grounded Theory, el análisis consideró un proceso de Codificación Abierta, donde las respuestas son categorizadas hasta alcanzar la saturación teórica, para luego realizar un análisis de Codificación Axial, que permite establecer relaciones entre las categorías obtenidas, reorganizando los datos para comprender el fenómeno, para finalmente, realizar un trabajo de Codificación selectiva, el cual permite la generación de un modelo explicativo del fenómeno observado.

González-Weil et al. (2009), Informe final proyecto FONIDE F310876.

El grado de competencia científica obtenido por los alumnos fue evidenciado a través de una Prueba construida ad hoc, basada en PISA 2006 y aplicada en el marco del proyecto FONIDE F310876. El nivel evaluado correspondió a tercero y cuarto de enseñanza media.

4 Las entrevistas de argumentos prácticos realizadas implicaron presentarle al profesor extractos audiovisuales de sus clases, para luego entrevistarlo acerca de las fundamentaciones que subyacen a su actuar en dicho episodio, de modo que el docente reflexiona respecto a su propio accionar en el aula (Gervais \& Correa, 2004). 


\section{RESULTADOS}

A continuación se exponen los resultados obtenidos a partir de los procesos de análisis antes mencionados.

\subsection{ANÁLISIS DESCRIPTIVO}

A partir del proceso de codificación abierta, se identificaron tres categorías centrales, las cuales dan respuesta a las preguntas que orientan esta investigación y que, como se verá a continuación, presentan determinadas subcategorías, propiedades y dimensiones:

(1) Secuencia Didáctica: Esta categoría, relacionada con la pregunta: ¿Qué actividades se realizan en la sala de clases y cómo se estructuran? presenta 5 subcategorías: (a) una actividad medular la cual está organizada, principalmente, en torno a experiencias de acceso directo al aprendizaje (Sanmartí, 2002), como el trabajo en laboratorio o salidas a terreno, las cuales contemplan la utilización de variados recursos, donde los alumnos son los protagonistas en la construcción del conocimiento; (b) momentos de la clase flexibles (inicio, desarrollo y final) los que se ajustan a las necesidades del alumno y se ponen al servicio del desarrollo de la actividad; (c) un ritmo activo de la clase, dado por un monitoreo y desplazamiento permanente del docente en el aula; (d) un proceso de orientación explícita de la actividad, donde el docente explica y reitera instrucciones, explicita los objetivos a alcanzar, supervisa el trabajo del alumnado y gestiona de manera eficiente el tiempo; (e) al término, el docente actúa como guía durante la clase, interactuando y retroalimentando a sus estudiantes.

(2) Competencia científica: Frente a la pregunta ¿Qué ámbitos de competencia científica aborda el docente en su clase? se reconoce la presencia de dos subcategorías: (a) promoción de conocimiento, capacidades y actitudes, las cuales se trabajan de manera interrelacionada. En relación al conocimiento, éste se construye en torno a conceptos científicos y, en menor medida, a la comprensión de la Naturaleza de las Ciencias. El ámbito de las capacidades está enfocado al trabajo de procedimientos característicos del quehacer científico como formular y resolver problemas. Como ámbito actitudinal se promueve, entre otros, el desarrollo de una actitud crítica y rigurosa y, en menor medida, la preocupación por el entorno. Respecto a (b) cómo se enseñan estas competencias, se observan dinámicas centradas en los alumnos, los cuales organizados de manera grupal y guiados por el docente, realizan experimentos, leen y resuelven problemas, entre otros.

(3) Interactividad: Esta categoría se relaciona con la pregunta ¿Qué características tiene la interacción profesor-alumno y de qué manera apoya esta interacción el aprendizaje?, y presenta 4 subcategorías: (a) presencia de un proceso activo y sistemático de negociación y construcción con los estudiantes, caracterizado por una relación simétrica en lo normativo entre el docente y los alumnos, diversos ciclos de interacción, lo que se ve posibilitado por la actitud de los estudiantes en relación al compromiso que presentan hacia el aprendizaje, y por el traspaso de autonomía desde el docente hacia el alumno a medida que transcurre la clase; (b) monitoreo intencionado y sistemático que realizan estos docentes durante la clase.; (c) andamiaje a partir de los requerimientos de los estudiantes, prestando apoyo pedagógico permanente durante toda la clase. (d) En última instancia, se observa la utilización recurrente de refuerzos sociales hacia los estudiantes para disponerlos positivamente al aprendizaje. 


\subsection{ANÁLISIS RELACIONAL}

A partir del análisis descriptivo de las prácticas, emergen dos fenómenos axiales, los cuales permiten comprender la relación que hay entre las categorías halladas anteriormente, $\mathrm{y}$, a partir del análisis de entrevistas, las condiciones que favorecen que el profesor realice las clases de la manera en que lo hace. Dichos fenómenos se enmarcan en un escenario global que presenta las siguientes condiciones intervinientes: relevancia de la alfabetización científica como objetivo de la Educación en Ciencias, la cual además de constituir un consenso internacional, es declarada por los Programas ministeriales chilenos y por los propios docentes del estudio; la presencia del paradigma constructivista en el discurso educativo y en la formación inicial, el cual contribuye a la idea de que es el mismo alumno quien construye sus conocimientos. Estrechamente relacionado a este paradigma se encuentra el hecho de que existe, particularmente a nivel internacional una promoción del enfoque indagatorio como estrategia de enseñanza, lo cual aparece como un elemento transversal en los programas de estudios actuales.

Una última condición interviniente, y que naturalmente incide sobre los procesos de enseñanza y aprendizaje es que nuestro país muestra una gran inequidad en el aprendizaje de las Ciencias en relación al nivel socioeconómico. Lo anterior fue claramente evidenciado en PISA 2006, donde Chile destaca por la alta incidencia del nivel socioeconómico en la explicación de resultados de desempeño de sus estudiantes (OECD, 2006). Esta condición claramente constituye un desafío para las prácticas de enseñanza de las ciencias a nivel secundario.

Así también es necesario señalar el contexto del estudio donde se producen estos fenómenos, el cual corresponde a: establecimientos urbanos de Enseñanza Media de la Región de Valparaíso (5 de la comuna de Viña del Mar, y 1 de la comuna de San Antonio), los que incluyen los tres tipos de dependencia (1 municipal, 4 subvencionados y 1 particular), con diversidad en la composición de género (mixtos (4), femenino (1) y masculino (1)). En relación al nivel educativo, éste corresponde a Tercer y Cuarto Año de Enseñanza Media. Por su parte, los docentes de este grupo, 2 damas y 4 varones, se caracterizan por presentar experiencia en Investigación en Ciencias y Ciencia Escolar, donde de los 6 docentes, 3 han participado en investigaciones científicas de su disciplina, ya sea durante su formación inicial o posterior y 4 han participado en proyectos de ciencia escolar ( 2 de ellos a través de la adjudicación de proyectos EXPLORA). Otro rasgo característico y exclusivo de estos docentes es el presentar estudios formales posteriores al pregrado en el ámbito pedagógico, tanto a nivel de Diplomado o Postítulo (2), Magíster (2), e incluso de Doctorado (1, en desarrollo). Por último, dos de los docentes del grupo han sido reconocidos por el MINEDUC con la Asignación de Excelencia Pedagógica (AEP). A continuación se describen los dos fenómenos encontrados.

\subsubsection{Fenómeno axial 1: Generación de actividades prácticas centradas en el alumno para favorecer el aprendizaje de la disciplina científica:}

"Si no se está diciendo que no se pase ese contenido o que el niño no aprenda ese contenido, el niño debe aprender ese contenido en función de problemas, muchas veces esa metodología, esa búsqueda de un problema para absorber un contenido es más rápida y eficiente" ( $P 1 /$ ECP 1/ 383-387) 
“...el hacer, significa que él (alumno) busque, investigue, vea un protocolo de acción, saque sus conclusiones y posteriormente elabore su hipótesis, luego vaya al laboratorio, ejecute, experimente y compruebe su hipótesis y concluya en función de lo que está observando" (S3) ECP 1/309-313)

La siguiente figura ilustra este primer fenómeno.

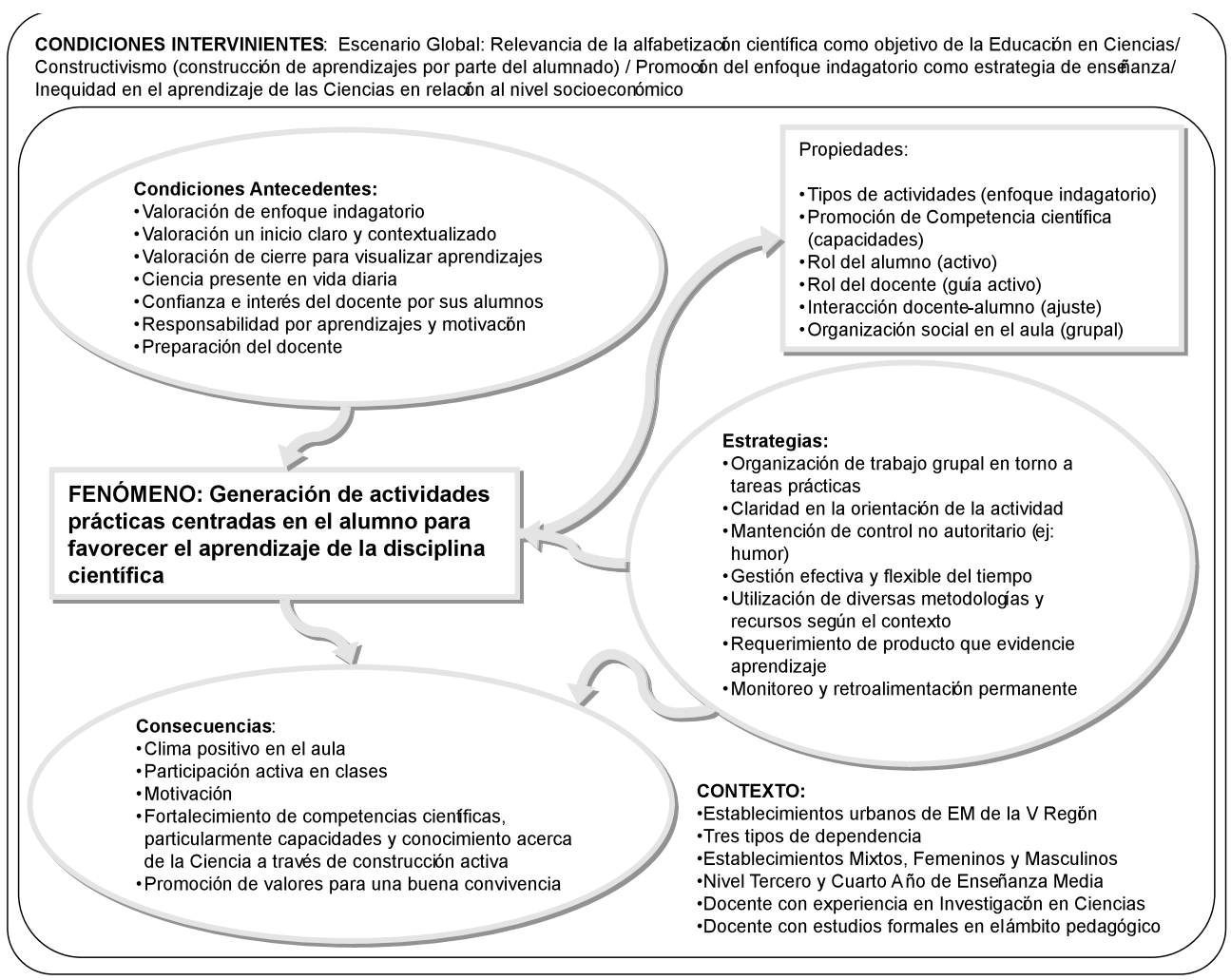

Figura 1. Fenómeno axial 1: Generación de actividades prácticas centradas en el alumno para favorecer el aprendizaje de la disciplina científica. Se señala entre paréntesis la dimensión más frecuente de las propiedades.

A partir del análisis realizado, identificamos como uno de los fenómenos distintivos de las prácticas de estos docentes, el hecho de que sus clases implican el desarrollo de una actividad práctica, la cual está centrada en la acción del alumno. Dentro de las propiedades que presenta este fenómeno, podemos identificar diversos tipos de actividades, que involucran diferentes niveles de autonomía en el alumno, las cuales incluyen desde lecturas, resolución de ejercicios y realización de experimentos, hasta expediciones científicas y situaciones de indagación abierta, en donde el alumno lleva a cabo pequeños 
procesos de investigación y los comunica a su comunidad a través de ferias y publicaciones. En relación a los ámbitos de competencia científica que se promueven en el marco de estas actividades, éstos se relacionan principalmente con el desarrollo de capacidades científicas, desde procesos científicos básicos (como observar y medir), intermedios (como interpretar y representar datos) hasta procesos más complejos, como formular hipótesis, crear diseños experimentales, sacar conclusiones y comunicarlas. Otros elementos que contribuyen a la promoción de competencias son la formulación y resolución de problemas por parte de los alumnos y la aplicación de lo aprendido a otros contextos. En cuanto a los conocimientos o contenidos conceptuales, los docentes promueven la construcción de conceptos científicos en el marco de su disciplina, de vocabulario científico asociado a dichos conceptos y, en menor medida, cierto conocimiento acerca de la Naturaleza de la Ciencia, en particular, en relación a cómo se genera investigación científica y, en algunos casos, a aspectos históricos de su desarrollo. Por otra parte, y en relación a las actitudes científicas, éste aspecto se ve menos desarrollado en las clases observadas, relacionándose básicamente con la promoción del cuidado del entorno y de sí mismo, y el presentar una actitud crítica y rigurosa frente al conocimiento.

Otra propiedad de este fenómeno es el rol del alumno, el cual es eminentemente activo, particularmente en la fase de desarrollo y cierre de las clases. El rol del docente por su parte es más bien de guía, pero no por ello menos activo que el alumno. Sus acciones se traducen a exponer y explicar contenidos, en un diálogo permanente con los alumnos, ejemplificando los conceptos con situaciones de la vida diaria y señalando la relevancia de su aprendizaje; formular preguntas de diferentes tipos, desde aquellas que demandan sólo recordar, hasta preguntas desafiantes, que requieren de elaboración y creatividad, así como servir de guía durante el desarrollo de la actividad por parte del alumno.

En cuanto a la interacción docente-alumno, se produce un ajuste, una situación de "apego", tanto en lo afectivo como en lo cognitivo, donde el docente muestra una flexibilidad que permite ajustar la actividad a las necesidades del alumno. Del mismo modo, el docente retroalimenta y monitorea los aprendizajes de manera permanente. Frente al error, el docente no es indiferente, y lo aborda señalando la respuesta correcta o bien, orientando y retroalimentando al alumno para llegar al concepto más adecuado.

El presente fenómeno se caracteriza por presentar cierta organización social de los alumnos, la cual va desde el trabajo individual, hasta el trabajo colaborativo con definición de roles, preponderando el trabajo grupal por sobre las demás formas sociales.

Respecto a las condiciones antecedentes que permiten explicar el surgimiento de este fenómeno encontramos una valoración del enfoque indagatorio en la enseñanza, como una herramienta para la promoción de capacidades, actitudes (particularmente valores), y conocimientos, así como fuente de motivación, que incluye la relación de la ciencia con la vida cotidiana y el aprendizaje a partir de problemas.

$P$ (dirigiéndose a los alumnos): "Bueno nosotros estamos haciendo la presentación de mini proyectos de aula, en la cual básicamente, se desarrolla el espíritu critico de parte de ustedes, a través de la indagación de algún procesos o fenómeno que tal vez ya esté, pero que nosotros aún no tenemos conocimientos de aquéllos a nivel de alumnos lo otro, dar la oportunidad de encontrar algunas técnicas, y que las cosas a veces están al lado de nosotros y no nos damos cuenta...." (S2, Clase 92, 154-163). 
Del mismo modo, la actividad indagatoria de los alumnos es valorada como instancia de comunicación entre los alumnos y de difusión a la comunidad, y en caso de las expediciones científicas, se consideran además como una experiencia de vida.

Así como los docentes valoran la existencia de una actividad indagatoria, que se desarrolla principalmente en la fase de desarrollo de la clase, una segunda condición antecedente es la valoración que hacen de las otras fases de la clase. Los docentes valoran un inicio claro y contextualizado de la clase, bajo el entendido de que si el alumno comprende lo que se va a realizar, habrá más posibilidad de lograr su interés. En relación al cierre, éste se ve como una instancia de evidenciación y monitoreo de aprendizajes, a través de la comunicación, ya sea oral o escrita, a la vez que una oportunidad para compartir significados, sintetizar y aplicar conocimiento.

Otra condición antecedente relevante es que los docentes presentan confianza e interés por los alumnos, refiriéndose en repetidas ocasiones a su necesidad de conocer las características personales de éstos.

"Llego al curso veo quiénes son, trato de alguna manera saber quiénes son, qué saben, qué no saben, cómo lo saben y ya captado ese, ese momento inicial cómo los chiquillos interactúan conmigo, entonces empiezo esta metodología... (S5/ ECP/81-84).

Este conocimiento se desarrolla a través del diálogo, como condición para el logro de un buen clima para el aprendizaje. Desde la perspectiva de este grupo de docentes, es este conocimiento el que permite la generación de vínculos afectivos con los alumnos, los cuales a la vez facilitan el aprendizaje.

Asociado con lo anterior, se encuentra el hecho de que estos docentes se sienten altamente responsables frente al aprendizaje y a la motivación de sus alumnos.

"Yo me noto al tiro cuando hay bajas notas, qué se yo hablo de bajas notas, ocho rojos, nueve rojos, hasta diez rojos puede llegar, yo me preocupo inmediatamente porque, o yo no estoy rindiendo, porque soy el que estoy enseñando, la alumna está disponible para mí para que ella aprenda y ella también vaya haciendo su propio conocimiento (M1/ ECP 3/313:317)

Lo anterior, determina que su enseñanza esté altamente centrada en el aprendizaje del alumnado.

Por otra parte, la preparación del docente, tanto en lo disciplinar como en lo pedagógico, es una condición que los profesores valoran altamente. Lo anterior, se evidencia en que todos presentan estudios formales (adicionales a los perfeccionamientos docentes) posteriores a su formación de pregrado (de postítulos a doctorado en curso).

Por último, estos docentes comprenden la Ciencia como una disciplina presente en la vida diaria, como necesaria y útil para la comprensión del entorno inmediato, la cual presenta una historia que no se debe desconocer.

En relación a las consecuencias que presenta el fenómeno en estudio podemos mencionar, en primer lugar, la generación de un clima positivo en el aula, el cual facilita la construcción de los aprendizajes, permite una participación activa en clases por parte del alumnado, así como la generación de una alta motivación por la clase. En consecuencia, los docentes de este grupo apenas si ocupan tiempo en llamar la atención o preocuparse por el comportamiento del grupo. Todo lo anterior, se condice con un fortalecimiento de competencias científicas, particularmente capacidades y conocimiento, tanto de como acerca de la Ciencia. Si bien las actitudes científicas se ven menos desarrolladas, sí se 
presenta promoción de valores para una buena convivencia, tales como la solidaridad, el respeto, la responsabilidad, el buen trato, justicia y preocupación por la comunidad.

Entre las estrategias que emplean los docentes para la mantención del fenómeno en estudio, podemos distinguir la presencia de una organización del trabajo grupal en torno a tareas prácticas, donde la actividad central de la clase se resuelve en grupos a través de la manipulación de materiales, resolución de ejercicios o realización de experimentos, entre otros. Este trabajo en ocasiones se da también de manera extra-escolar, y a lo largo de un tiempo más prolongado, al final del cual los alumnos exponen sus resultados y conclusiones frente al grupo-curso o frente a la comunidad escolar. Otra estrategia utilizada por el docente es ir generando particularmente al inicio, pero también durante toda la clase, una claridad en la orientación de la actividad que el alumno ha de realizar, lo que logra a través de la contextualización, explicitación y repetición de objetivos e instrucciones, estrategia que es justificada por el docente en la medida en que orienta y produce interés en el alumnado.

En relación a la ya mencionada generación de un clima positivo en el aula como consecuencia de las acciones del docente, una de las estrategias empleadas es la mantención de un control no autoritario, donde el diálogo es valorado por sobre la represión. A su vez, una estrategia recurrente en el grupo de docentes observados es la gestión efectiva y flexible del tiempo. La mayoría de los docentes se preocupa de cumplir con los tiempos dispuestos para las diferentes actividades de la clase. No obstante lo anterior, éstas planificaciones son flexibles y se ajustan a las necesidades de los alumnos y a la actividad realizada.

Otra estrategia desarrollada por los docentes dice relación con la utilización de diversas metodologías y recursos según el contexto.

"Uno debe tener una amplia gama de metodologías de trabajo para esa misma planificación, colocando las alternativas correspondientes porque si no está resultando de esa manera tiene que irse a otra manera, por supuesto que la estructura es casi siempre la misma, y eso es lo que no debe aburrir a los alumnos que ya le pesquen la metodología de trabajo, aquí debe haber una diversidad de cosas (M1/ ECP 3/ 245-151).

En lo que se refiere al aseguramiento de los aprendizajes, los docentes utilizan como estrategia, normalmente al final de la clase, el requerimiento de un producto que evidencie aprendizajes. Éste a menudo se expresa en un informe de laboratorio, la resolución de una guía o la comunicación oral de resultados, producto que normalmente es calificado. Cabe decir que, durante el desarrollo de la actividad, los docentes en estudio se caracterizan por realizar un monitoreo y retroalimentación permanente, particularmente a través de preguntas. Este aspecto constituye una de las características centrales del segundo fenómeno identificado en este estudio.

\subsubsection{Fenómeno axial 2: Proceso activo y sistemático de negociación y construcción de} competencia científica

\footnotetext{
A3:Y en ésta, ¿podemos concluir que a menos tiempo menos vueltas?

P: A ver, ¿qué pusieron como hipótesis? (mira la hoja) no han puesto la hipótesis ahí, a ver, ¿cuánto fue el $R$ ?.

A2: 28

P: $28 \mathrm{~cm}$. ¿Qué hubiera pasado si en vez de 28 hubiese sido 56?
} 
A3: Entre más radio, menos vueltas

$P$ : Ahí está la respuesta

A2: $Y$ entre menos radio, más vueltas

P: Si pues, con eso puedes concluir

$(M 1 / 6 / 540-548)$

La siguiente Figura ilustra este segundo fenómeno:

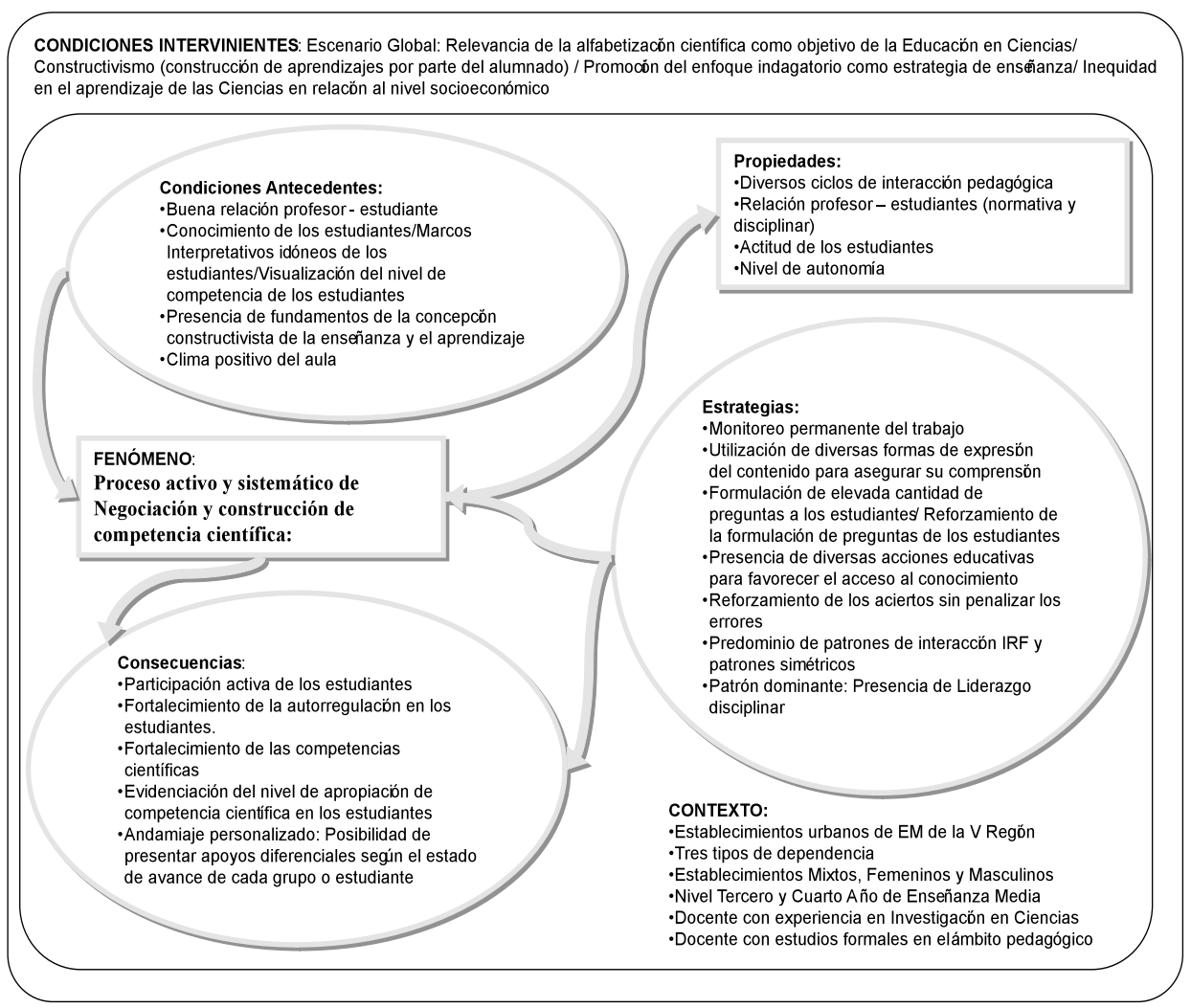

Figura 2. Fenómeno axial 2: Proceso activo y sistemático de Negociación y construcción de conocimientos científicos.

A partir del análisis realizado identificamos como segundo fenómeno axial, el que estos docentes realizan un proceso activo y sistemático de negociación y construcción de competencia científica (ver Figura 2). Dentro de las propiedades que presenta este proceso de construcción destacan: (a) la presencia sistemática de ciclos de interacción pedagógica con los estudiantes, los cuales se caracterizan por ser diversos dependiendo de los requerimientos planteados por los estudiantes y por lograr establecer patrones abiertos en el que se solicita a los estudiantes respuestas que requieren elevados nivel de 
elaboración. (b) La relación profesor-estudiantes que se establece durante este proceso, donde encontramos predominio de una interacción simétrica en lo normativo (monitoreada por el docente) y más vertical en el ámbito disciplinar en la lógica de relación profesor experto/estudiantes novatos, esta última se va transformando a medida que los estudiantes adquieren mayor dominio del tema. (c) Actitud de los estudiantes durante dicho proceso, donde encontramos una gradiente que va desde una disposición favorable hasta una actitud comprometida y activa de los estudiantes. (d) Nivel de autonomía de los estudiantes en esta construcción, que va desde el traspaso de control en aspectos más básicos (instrumentales), hasta el traspaso de control en aspectos medulares/fundamentales, presentándose especialmente en momentos más avanzados de la clase. Algunas de estas propiedades se aprecian en la siguiente cita:

P: Ya chiquillas, ¿quiénes son aquí? (le van diciendo los apellidos y ella los anota)

A8: Profesora, no sabemos qué sistema es, si es cerrado o aislado.

$P:$ ¿Qué creen ustedes?

A9: Cerrado

$P:$ ¿Por qué, cual es la diferencia? ¿Cuál es la definición de uno y de otro?

A8: Cerrado es cuando hay intercambio de calor y no de masa, y aislado es cuando no hay intercambio ni de calor ni de masa

$P:$ ¿Y que sucedió en el experimento?

(S2/92/79-89)

Respecto a las condiciones antecedentes que permiten explicar el surgimiento de este fenómeno, encontramos como elemento esencial los fundamentos o creencias que sustentan las prácticas de estos docentes entre las cuales encontramos (a) elevado conocimiento de los estudiantes respecto a: su historia personal, las capacidades cognitivas que presentan, así como respecto a la personalidad de éstos. Esto les otorga un marco interpretativo idóneo al momento de construir conocimiento, ya que les permite visualizar el nivel de competencias de sus estudiantes. (b) Presencia de fundamentos de la concepción constructivista de la enseñanza y el aprendizaje. Estos profesores consideran a los estudiantes protagonistas activos del proceso educativo y reconocen la diversidad de estudiantes y contextos en que se produce el fenómeno del aprendizaje (c) el contar con un Clima Positivo en el aula, el cual permite un ambiente de confianza que favorece el proceso activo y sistemático de construcción de conocimiento científico. En definitiva, la valoración de la indagación, aspecto que fue abordado anteriormente, impregna la construcción de conocimiento que se desarrolla en la clase de ciencias de estos docentes.

"el primero y más grave de todos los errores en la escolaridad o escolarización es que el profesor y relacionado con lo que conversábamos, viene intencionado a hacer su clase, o sea tiene una intención prediseñada en su cabeza y entra por la puerta y la ejecuta, el alumno se da cuenta de esa intención cuando trata de llevarlo hacia otros ámbitos o otras preguntas y el profesor pesca el timón y lo agarra con fuerza y lo centra en, en lo que él pretende hacer en la clase, esa, esa intencionalidad, es el, a mi juicio, el principio de la escolarización, el profesor viene a responderle a los alumnos preguntas que ellos todavía no se hacen y ahí mataste, mataste el fenómeno pedagógico o la posibilidad de aprendizaje porque (cambia la voz) niños hoy día vamos a ver fracciones, las fracciones se crearon en la edad, que se yo, los griegos, los griegos cuando dividian...(vuelve a voz normal)" (ECP P1/1/128:140). 
En cuanto a las consecuencias que presenta este fenómeno, podemos mencionar: (a) la participación activa de los estudiantes en clases, quiénes asumen un rol protagónico, fortaleciendo con ello la autorregulación de sus procesos de aprendizaje; (b) impacto en el fortalecimiento de las competencias científicas, especialmente en lo referido a las capacidades y la apropiación de los conocimientos disciplinares y más discretamente en las actitudes hacia la ciencia; (c) que este fenómeno posibilita que el docente evidencie el nivel de apropiación de capacidades y conocimientos científicos en los estudiantes y del grupo curso, lo que les permite prestar apoyos diferenciales según el estado de avance de los estudiantes o grupos realizando un andamiaje ajustado a los requerimientos de los estudiantes.

Respecto a las estrategias que despliegan estos docentes para mantener la presencia del fenómeno, encontramos: (a) Monitoreo permanente del trabajo de los grupos y de los estudiantes. Este monitoreo se realiza durante toda la actividad y en distintos niveles y ámbitos. Va desde la revisión de la comprensión de las instrucciones, al aprendizaje de competencias (previas y de la clase), y del progreso de la actividad hasta la convivencia en el aula.

(referido al monitoreo) "ah, es fundamental porque así yo me voy dando cuenta si van, si les va quedando como claro lo que se ha estudiado la semana anterior, como decía, me parece que hicimos un laboratorio antes, y... y así me voy dando cuenta como, como va la, cómo van aprendiendo, cómo van recordando aquello que vimos..." (S3, EAP, 1178-1181)

(b) Elevada cantidad de preguntas durante la clase, las que además son diversas, tanto en el nivel cognitivo que conllevan (recordar, comprender, aplicar y evaluar) como en el tipo de conocimiento que solicitan (factual, conceptual, procedimental). Esto, sumado a que estos profesores incitan a los estudiantes a formular preguntas, genera un ambiente de diálogo, análisis y reflexión permanente; (c) las acciones educativas implementadas por los docentes para favorecer la construcción de competencias, que implican entregar pistas de información de manera gradual, el repetir ideas fundamentales, elaborar a partir de ideas de los estudiantes, recapitular ideas (síntesis), utilizar sus propias experiencias, presentar objetos, y establecer relaciones con la vida cotidiana; (d) Diversas formas para representar el contenido: Oral, escrita, gráfica y sensoriomotriz, asegurando de esta manera una adecuada comprensión. Estos recursos están sustentados en las creencias de los profesores respecto a la necesidad de diversificar las formas de trabajo en función de los aprendizajes de los estudiantes; (e) los docentes destacan positivamente las acciones e ideas de los estudiantes a través de distintas formas: verbal, gestos, etc. El destinatario puede ser individual o grupal, y el fundamento que utilizan para realizarlo se basa en la valoración del trabajo y la valoración de la persona. Respecto a la forma puede ser una frase sencilla sin justificación o una frase justificada. (f) Estos docentes valoran que los estudiantes opinen en clases por sobre la calidad y exactitud de la opinión, naturalizando de este modo la posibilidad de equivocarse como parte sustancial del proceso de aprendizaje. Respecto a los errores, si bien estos no son penalizados, el docente recurre a diversas secuencias pedagógicas para su corrección, acciones que van desde la corrección directa (entregando la respuesta correcta), formulación o reformulación de preguntas y/o a través de una secuencia colateral con preguntas que posibiliten a los estudiantes encontrar las evidencias necesarias para identificar el error, y de esta manera llegar a la respuesta de manera constructiva. 


\subsection{ANÁLISIS SELECTIVO}

A partir de los análisis realizados y de los objetivos perseguidos por la investigación, elaboramos un modelo explicativo o fenómeno central. Dicho modelo posibilita por un lado, la comprensión de las prácticas innovadoras que presentan los docentes del estudio, y por otro, abre interesante posibilidades de intervenir en la transformación de las prácticas de otros docentes, en pos del fortalecimiento de competencia científica en el alumnado. A la vez, el fenómeno descrito a continuación, nos da orientaciones acerca de la formación inicial de docentes secundarios de ciencia y la docencia universitaria asociada a esta formación.

\subsubsection{Fenómeno central: Indagación como reflexión sobre la propia} práctica durante el desarrollo de la clase

A partir de los antecedentes recabados podemos distinguir como fenómeno central la indagación como reflexión sobre la propia práctica durante el desarrollo de la clase (Figura 3).

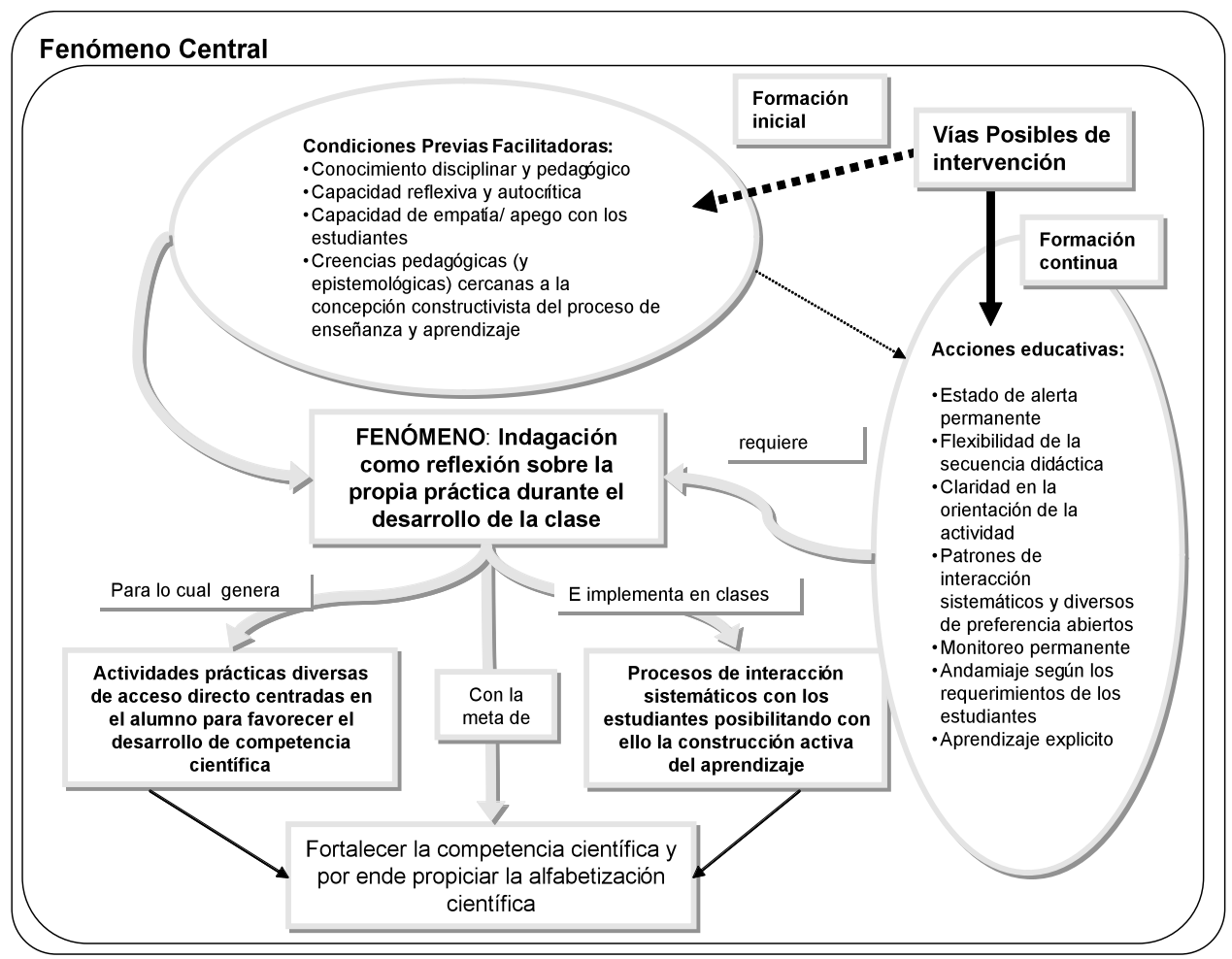

Figura 3. Fenómeno central: Indagación como reflexión sobre la propia práctica durante el desarrollo de la clase 
Ésta se caracteriza por un cierto "estado de alerta permanente" durante el desarrollo de la clase, análogamente a un juego de ajedrez, donde el docente está atento a los alumnos particulares, pero sin perder de vista el grupo-curso, y actúa a la vez que analiza, anticipa y reflexiona acerca de su acción y sus consecuencias, promoviendo procesos de indagación y fortaleciendo el desarrollo de competencias científicas en sus alumnos. Este estado de alerta, así como las "jugadas estratégicas" que realiza el docente para mantener la atención, fomentar la motivación y potenciar el desarrollo de competencias, es facilitado además, por el alto conocimiento que mantiene el docente de sus alumnos.

Esta indagación, como reflexión durante la práctica se concreta en la planificación e implementación de actividades prácticas diversas que se constituyen como experiencias de acceso directo al aprendizaje, en el que los alumnos son los protagonistas en la construcción de su competencia científica.

Los docentes cuyas prácticas se describen en este estudio, comprenden que sus alumnos son diversos, y que la enseñanza debe estar orientada por las características del alumnado, y sus maneras de aprender, lo cual se relaciona con concepciones pedagógicas más constructivistas. Esto implica que las actividades que se han de planificar, consideran los intereses y dificultades del alumnado, son flexibles y se van ajustando a sus necesidades.

Adicionalmente, estas actividades se caracterizan porque es el alumno quien realiza la acción, y en diferentes grados, la crea, la comparte y la comunica. Lo anterior no significa que el docente tenga un rol menos activo. El docente en las clases observadas constituye un guía activo, que orienta y monitorea los procesos de aprendizaje, orientando el desarrollo de competencias científicas en sus alumnos. Aunque en diferente medida, las prácticas observadas presentan una alta componente de indagación, donde se expone al alumno a problemas y tareas que demandan diferentes capacidades científicas, como la formulación y justificación de hipótesis, el diseño de experimentos, la puesta en marcha de actividades experimentales y la discusión y comunicación de resultados, promoviéndose en las clases observadas preferentemente capacidades asociadas al quehacer científico. Complementariamente, se promueve la comprensión de conceptos científicos, y en menor medida, actitudes relacionadas con la Ciencia. Todo lo anterior se relaciona con un cierto modelaje que realiza el docente del quehacer científico, expresado en los casos más explícitos, en pequeñas investigaciones o indagaciones abiertas que los alumnos desarrollan de manera relativamente autónoma y que son luego comunicadas a la comunidad escolar.

Por otra parte, uno de los aspectos más relevantes que emerge de los resultados insta a que los docentes sean capaces de reflexionar (de manera explícita) acerca de sus prácticas pedagógicas durante el momento de la enseñanza, de manera de poder ajustarlas según los requerimientos de los estudiantes. Pozo (2003) señala que para lograr adquirir conocimiento es necesario hacer explícito el aprendizaje, es decir, en el caso de este estudio, lograr que los docentes hagan concientes dichas prácticas durante la enseñanza, re-presentando (redescribiendo), a través de otra representación cognitiva (tal como el lenguaje) aquello que es implícito (Pozo, 2003).

Estos docentes logran explicitar sus prácticas, debido al proceso de interacción y diálogo sistemático que establecen con sus estudiantes. Dicho diálogo les permite visualizar cómo se está dando la construcción del aprendizaje y hacer los ajustes necesarios, reformulando sus prácticas. A través de la exploración de las concepciones docentes, se evidencia la presencia de un diálogo interno permanente durante la clase en pos del aprendizaje de calidad de sus estudiantes. 
Condiciones previas facilitadoras que permiten el fenómeno central, y que, por lo tanto, forman parte del modelo, son: (a) un mayor grado de conocimiento disciplinar (dado por ejemplo, por experiencias en investigación científica), pedagógico y didáctico (dados principalmente por estudios formales posteriores al pregrado o la participación en programas de educación en ciencias), lo que trae como consecuencia (b) creencias pedagógicas y epistemológicas cercanas a visiones más constructivistas (c) una cierta capacidad de empatía y apego con sus estudiantes, lo que facilita el conocimiento que los docentes presentan de sus alumnos y (d) la gran capacidad reflexiva y autocrítica de los docentes, lo cual constituiría el motor de sus innovaciones pedagógicas y la capacidad de ajuste al alumnado.

En relación a las acciones educativas que expresan y mantienen el fenómeno central de indagación reflexiva en la práctica, consideramos que las más relevantes, mencionadas anteriormente, son: el estado de alerta permanente, la flexibilidad de la secuencia didáctica (en cuanto a duración y orientación de los momentos de la clase, que permite ajustarse a los alumnos); la claridad en la orientación de la tarea, los patrones de interacción sistemáticos y diversos de preferencia abiertos, el monitoreo permanente, el andamiaje según los requerimientos de los estudiantes y finalmente, el aprendizaje explícito que hacen los docentes de su propia experiencia docente, a través del ejercicio de la reflexión sobre y en la práctica, la cual además se apoya en insumos, como la propia opinión de los alumnos y sus resultados de aprendizaje.

Tanto las condiciones previas facilitadoras, como las acciones educativas, son señaladas como vías posibles de intervención, en el entendido de que podrían constituir la puerta de entrada para la intervención de las prácticas de otros docentes, tanto en el marco de la formación inicial (particularmente, apuntando a la generación de las condiciones previas), como a la formación continua, a través de la reflexión sobre las acciones educativas, y la inclusión de ellas en las prácticas de los docentes ya en ejercicio.

\section{DISCUSIÓN}

El presente estudio contribuye con evidencia local a los actuales modelos de enseñanza secundaria en ciencias, los cuales son eminentemente teóricos y a menudo demasiado abstractos para imaginarlos en acción. En segundo lugar, reafirma el valor de la reflexión docente como elemento clave para la pertinencia de las prácticas, a la vez que ilustra de qué manera esta reflexión se manifiesta también en el propio transcurso de la clase. En tercer lugar, da ciertas luces acerca de las características que podría tener la indagación científica en las aulas secundarias, particularmente en relación a las condiciones bajo las cuales es posible la construcción de conocimientos y la generación de competencia científica a través de procesos indagatorios que exploran fenómenos de la naturaleza. Por último, nos permite extrapolar el proceso de indagación y construcción de conocimientos más allá del aula, donde -así como el alumno construye conocimiento científico a partir de la problematización de fenómenos naturales- su profesor construye conocimiento pedagógico y didáctico, a través de la indagación y análisis de las propias prácticas, lo que le permite ir ajustando su enseñanza al requerimiento del alumnado incluso en el propio desarrollo de la clase. Aunque el proceso descrito no necesariamente ha sido explicitado y sistematizado por todos los docentes del estudio, lo reconocemos como un 
elemento común al conjunto de profesores participantes. Este mismo proceso de reflexión es el que ha permitido a los docentes reconocer cuáles son sus necesidades de desarrollo profesional, lo que los ha llevado a formarse en ámbitos pedagógicos, disciplinares y didácticos, aun después de su formación inicial. Por último, y si los docentes llegaran a un proceso de indagación/reflexión sistemático de sus prácticas, podríamos relacionar lo observado con el concepto de indagación científica como enfoque pedagógico, entendiendo ésta como un proceso reflexivo de exploración, planificación, comunicación, construcción, y re-construcción del quehacer docente, el cual constituye una espiral de mejora continua del ejercicio profesional.

\section{IMPLICANCIAS PARA LA FORMACIÓN INICIAL Y CONTINUA DE DOCENTES DE CIENCIA SECUNDARIOS}

A partir de los resultados, podemos proponer que, tanto en la formación inicial como continua de docentes, la reflexión crítica del propio quehacer docente, debiera ser el eje central. Si bien esta idea no es nueva, generalmente estas reflexiones se realizan sobre la práctica y en solitario. Nuestros resultados abogan adicionalmente por una reflexión sobre, pero, especialmente, durante la práctica, la cual, si se considera la indagación científica como enfoque pedagógico, debiera darse además en un marco social de reflexión con otros actores (como otros docentes), sobretodo con los propios alumnos. Iniciativas de formación profesional como los Estudios de Clase Japonés (Isoda et al., 2007), el uso de bitácoras o diarios de clase, la auto-observación de prácticas, la diversificación de los patrones de interacción con los estudiantes en la construcción de competencia científica, entre otros, son ejemplos que promueven la reflexión sobre el quehacer docente, tanto a nivel individual como social.

Un segundo aspecto a abordar, particularmente en la formación docente inicial, es el reforzamiento del conocimiento disciplinar (incluyendo conocimiento acerca de la Ciencia, por ejemplo a través de vivenciar procesos de investigación científica y hacerlos explícitos), además del conocimiento pedagógico y didáctico, lo que a su vez incidiría sobre las creencias epistemológicas y pedagógicas.

Finalmente, el abordar el aprendizaje de las acciones educativas desplegadas por los docentes en estudio, incluyendo el aprendizaje explícito sobre y durante sus prácticas, puede ser especialmente relevante para los modelos de formación continua, los cualesconsiderando lo fundamental que resulta desde la evidencia aquí presentada la indagación de los propios procesos de enseñanza- proponemos podrían darse en un proceso de investigación- acción colaborativa, donde el docente, considerando la inercia que puede traer su ejercicio profesional prolongado en el tiempo comience por la problematización, recoja evidencia, reflexione, comparta con otros y en definitiva transforme gradualmente sus prácticas.

Cabe preguntarse, para terminar, ¿Cómo se produce la transformación de las prácticas de un profesor de ciencias a través de una investigación- acción? Y, particularmente, ¿cómo ello puede llegar a constituirse en un modelo de desarrollo profesional efectivo? Preguntas que quedan abiertas y que invitamos a explorar. 


\section{REFERENCIAS BIBLIOGRÁFICAS}

Abell, S., D. Smith \& Volkmann, M. (2006). Inquiry in Science Teacher Education. En Flick, L y N. Lederman (Eds.), Scientific inquiry and the nature of science: Implications for teaching, learning, and teacher education (pp. 389-425). Springer: Netherlands.

Albertini, R., G. Cárdenas-Jirón, J. Babel, G. Díaz Véliz, J. Eyzaguirre, A. Labra y R. Lewin (2005). Enseñanza de las ciencias a nivel escolar y formación en ciencia en el pregrado universitario. En Ureta, T., J. Babul, S. Martínez y J. Allende. Análisis y Proyecciones de la Ciencia Chilena 2005. Academia de Ciencias. Recuperado el 03-04-08 de: http://www. academia-ciencias.cl/?module=investig.

Alberts, B. (2008). Considering Science Education (editorial). Science, 319, 21, 1189.

Chacón (2003). Calidad y Equidad en la Educación Media. Santiago de Chile:Depesex/Bcn/Serie Informes, Año XIV, N ${ }^{\circ}$ 129. Recuperado el 07-08-07 de: http://www.bcn.cl/bibliodigital/pben/ informes/estudios_pdf_informes/nro129.pdf

Devés, R. (2004) "Metodología de la enseñanza de las ciencias: visión de un científico". Revista Extramuros, n.2. Recuperado el 17-07-08 de: http://www.umce.cl/revistas/extramuros/extramuros_n02_a05.html

Gervais, C. \& Correa, E. (2004). Explicitación del saber de experiencia de los profesores en el contexto de las prácticas docentes: un marco conceptual y metodológico. Íkala, 9 (15), 141 - 167

Gil-Pérez, D. y Vilches, A. (2001). Una alfabetización científica para el siglo XXI. Obstáculos y propuestas de actuación. Investigación en la Escuela, 43, 27-37.

González-Weil, C., Martínez, M.; Martínez, C.; Cuevas, K \& Muñoz, L. (2009). La Educación Científica como apoyo a la Movilidad Social: Desafíos en torno al rol del Profesor Secundario en la Implementación de la Indagación Científica como Enfoque Pedagógico". Estudios Pedagógicos, 35, 1, 63-78

Isoda, M. Arcavi, A. y Mena, A. (2007). El estudio de clase Japonés en Matemáticas: su importancia para el mejoramiento de los aprendizajes en el escenario global. Valparaíso: Ediciones Universitarias de Valparaíso.

Láscaris, T. (2008). Legitimación social de la ciencia como factor estratégico para el desarrollo en América Latina. Intervención en el Congreso Iberoamericano Ciudadanía y Políticas Públicas en Ciencia y Tecnología, Madrid 5 al 8 de Febrero de 2008.

Macedo, B. y Katzkowicz, R. (2005). Alfabetización científica y tecnológica: Aportes para la reflexión. Publicación digital de OREALC/UNESCO Santiago. Recuperado el 03/05/06 de: http://www.unesco.cl/medios/alfabetizacion_cientifica_tecnologica_aportes_reflexion.pdf

OECD (2006). Assessing Scientific, Reading and Mathematical Literacy: A framework for PISA 2006. Recuperado el 20-12-06 de http://www.oecd.org/dataoecd/63/35/37464175.pdf

Pasmanik V., Diana y Cerón F., Raúl (2005). Las prácticas pedagógicas en el aula como punto de partida para el análisis del proceso enseñanza-aprendizaje: un estudio de caso en la asignatura de química. Estudios Pedagógicos, 31, 2, 71-87.

Pozo, J. (2003). Adquisición de conocimiento. Madrid: Ediciones Morata.

Programa ECBI (2007). En Internet bajo: www.ecbichile.cl. Recuperado el 20-03-07

Reimí, M. (2002).La investigación científica y el desarrollo tecnológico, reflexiones para la sociedad latinoamericana. Ciencia y Sociedad, 27, 4, 549- 555

Sanmartí, N. (2002). Didáctica de las Ciencias en la Educación Secundaria Obligatoria. Madrid: Síntesis.

Strauss, A. \& Corbin, J. (1990). Basics of qualitative research: Grounded theory procedures and techniques. London: Sage.

Windschitl, M. (2003). Inquiry Projects in Science Teacher Education: What Can Investigative Experiences Reveal About Teacher Thinking and Eventual Classroom Practice? Science Education, 87, 112-143. 